Andrew F. Hundley • Morton B. Brown •

Linda Brubaker - Geoffrey W. Cundiff - Karl Kreder •

Peter Lotze • Holly E. Richter • Halina Zyczynski •

Anne M. Weber - Anthony G. Visco

\title{
A multicentered comparison of measurements obtained with microtip and external water pressure transducers
}

Received: 6 May 2005 / Accepted: 18 September 2005

(C) International Urogynecology Journal 2005

\begin{abstract}
This study compared simultaneous intravesical pressure readings obtained with catheter-mounted microtip transducers and external water pressure transducer catheters during filling cystometry. Women undergoing multichannel urodynamic testing were randomly assigned to one of three groups: two microtip catheters, two external water pressure transducer catheters, or one of each type. Intravesical pressure was measured simultaneously for each transducer combination in each subject for minimal and maximal Valsalva effort and minimal, moderate, and maximal cough effort at two sequential bladder volumes $(150$ and $300 \mathrm{ml})$. Paired $t$ tests were used to compare the means of the intravesical pressure obtained by the two types of catheters. The largest mean dif-
\end{abstract}

Abstract presented at the International Continence Society Annual Meeting, Paris, France, August, 2004

A. F. Hundley · A. G. Visco

Division of Female Pelvic Medicine

and Reconstructive Pelvic Surgery,

University of North Carolina at Chapel Hill,

Chapel Hill, NC, USA

M. B. Brown

Pelvic Floor Disorders Network

Data Coordinating Center, University of Michigan,

Ann Arbor, MI, USA

L. Brubaker

Division of Female Pelvic Medicine

and Reconstructive Pelvic Surgery,

Loyola University,

Maywood, IL, USA

G. W. Cundiff

Division of Female Pelvic Medicine

and Reconstructive Pelvic Surgery,

Johns Hopkins Bayview Medical Center,

Baltimore, MD, USA

K. Kreder

Division of Female Pelvic Medicine

and Reconstructive Pelvic Surgery,

University of Iowa,

Iowa City, IA, USA ferences were observed when comparing microtip and water pressure transducers. Correlations of maximum pressure were consistently high between two microtip transducers and two water pressure transducers but lower for the microtip-water combination. Excellent reproducibility was demonstrated with transducers of similar types for intravesical pressures recorded during Valsalva and cough in women without prolapse. However, considerable variability was seen in pressures recorded by different transducers, particularly dependent on the water catheter manufacturer, indicating that intravesical pressure recordings from microtip and water-based systems are not interchangeable.

Keywords Microtip - Pressure transducer - Cough pressure $\cdot$ Valsalva $\cdot$ Correlation

\section{P. Lotze}

Division of Female Pelvic Medicine

and Reconstructive Pelvic Surgery,

Baylor College of Medicine,

Houston, TX, USA

H. E. Richter

University of Alabama at Birmingham

Continence Center,

Birmingham, AL, USA

\section{H. Zyczynski}

Division of Female Pelvic Medicine

and Reconstructive Pelvic Surgery,

Magee Women's Hospital,

Pittsburgh, PA, USA

\section{A. M. Weber}

National Institute of Child Health

and Human Development,

Bethesda, MD, USA

A. G. Visco $(\bowtie)$

Department of Obstetrics and Gynecology,

University of North Carolina at Chapel Hill,

Chapel Hill, NC, USA

Tel.: +1-919-9664717

Fax: +1-919-8439952 


\section{Introduction}

Abdominal leak point pressure (LPP) is defined as the intravesical pressure at which urine leakage occurs due to increased abdominal pressure in the absence of a detrusor contraction [1]. LPP is commonly recorded during filling cystometry as part of the assessment of urinary incontinence in women. However, LPP is calculated in different ways [25], usually either as the total pressure or as the change over baseline. To ensure that LPP is defined clearly in articles that report it, the International Continence Society (ICS) recommends describing LPP according to the site of measurement (rectal, vaginal, or intravesical) and the method by which pressure is generated (cough or Valsalva). In addition, ICS recommends that investigators explicitly state whether the absolute value or the change in pressure from baseline is recorded for LPP [3].

The two most commonly used systems to measure pressures during urodynamic evaluation are catheter-mounted microtip and external water pressure transducer systems. Microtip and water-based systems have been compared for urethral pressure profiles [6] but not for LPPs. For clinical and research purposes, it is necessary to know whether pressures obtained with different systems are similar enough to be used interchangeably.

This has important implications in patient care for clinicians who recommend different treatments based on the severity of urethral dysfunction in women with urinary incontinence. Implications for research are also important, particularly for multicenter trials that include urodynamic data where different centers may use different transducer systems.

In a previous study using an experimental model, simultaneous pressure readings were compared using microtip and water perfusion catheter systems [7]. This model utilized a partially filled, 1,000-cc latex intravenous fluid bag to serve as a simulated bladder. Microtip and water perfusion catheters were then inserted through the access ports at the base of the bag. External manual compression of this system simulated the increased intra-abdominal pressure of "coughs" and "Valsalvas" of different strengths at different filling volumes. Correlation between the two systems was very high $(r=0.99)$.

The purpose of this study was to determine the degree of correlation between catheter-mounted microtip transducers and external water pressure transducers when measuring Valsalva and cough LPP in women undergoing urodynamic testing.

\section{Materials and methods}

All terminology follows ICS standards [8]. The study population consisted of 107 women, 21 years of age or older, referred for urodynamic testing for the assessment of urinary incontinence or other urinary complaints at one of the five Pelvic Floor Disorders Network (PFDN) clinical centers. Women were excluded for known genitourinary anomalies, stage III or IV pelvic organ prolapse, pregnancy, or inability to complete urodynamic testing. Women were enrolled between October 2003 and February 2004 following approval by the Institutional Review Board at each participating site. Women referred for urodynamic testing at these sites were approached for study participation. Individual site enrollment ranged from $12-32 \%$ of the total study population. After obtaining written informed consent, women were randomly assigned to one of the three transducer combinations: two microtip transducers [microtip-microtip (M-M)], two external water pressure transducers [water-water $(\mathrm{W}-\mathrm{W})]$, or one microtip and one water transducer [microtip-water $(\mathrm{M}-\mathrm{W})]$. Assignment was performed by block randomization in a 1:1:3 ratio with a masked block size of 5 using sequentially numbered, sealed, opaque envelopes. Patients were assigned unique identifying
Table 1 Demographic characteristics and testing conditions for 95 women undergoing filling cystometry with transducer pairs

$M-M$ Microtip-microtip transducer combination, $M-W$ microtip-external water pressure transducer combination, $W-W$ external water-external water pressure transducer combination ${ }^{a}$ Reported as mean \pm standard deviation

${ }^{\mathrm{b}}$ Reported as number (percentage)

\begin{tabular}{|c|c|c|c|c|}
\hline & \multicolumn{4}{|c|}{ Study Group } \\
\hline & $\mathrm{M}-\mathrm{M}$ & $\mathrm{M}-\mathrm{W}$ & $\mathrm{W}-\mathrm{W}$ & All Subjects \\
\hline$n$ & 15 & 62 & 18 & 95 \\
\hline Age $(\text { years })^{\mathrm{a}}$ & $55.5 \pm 13.2$ & $54.1 \pm 12.9$ & $53.1 \pm 14.1$ & $54.2 \pm 13.0$ \\
\hline Height $(\mathrm{cm})^{\mathrm{a}}$ & $166.1 \pm 6.6$ & $163.4 \pm 6.5$ & $164.7 \pm 8.7$ & $164.1 \pm 6.9$ \\
\hline Weight $(\mathrm{kg})^{\mathrm{a}}$ & $79.7 \pm 20.7$ & $77.9 \pm 17.4$ & $83.9 \pm 28.1$ & $79.2 \pm 19.8$ \\
\hline BMI $\left(\mathrm{kg} / \mathrm{m}^{2}\right)^{\mathrm{a}}$ & $29.0 \pm 7.6$ & $29.2 \pm 6.2$ & $30.9 \pm 9.1$ & $29.4 \pm 6.9$ \\
\hline \multicolumn{5}{|l|}{ Race $^{\mathrm{b}}$} \\
\hline Black & $3(19)$ & $5(8)$ & $1(6)$ & $9(9)$ \\
\hline Multiracial & $0(0)$ & $1(2)$ & $0(0)$ & $1(1)$ \\
\hline White & $13(81)$ & $57(90)$ & $15(94)$ & $85(89)$ \\
\hline Hispanic & $0(0)$ & $1(2)$ & $0(0)$ & $1(1)$ \\
\hline \multicolumn{5}{|c|}{ Exam Positioning ${ }^{\mathrm{b}}$} \\
\hline Standing & $0(0)$ & $7(11)$ & $5(31)$ & $12(13)$ \\
\hline $45^{\circ}$ Sitting & $16(100)$ & $56(89)$ & $11(69)$ & $83(87)$ \\
\hline \multicolumn{5}{|l|}{ Fluid Used ${ }^{\mathrm{b}}$} \\
\hline Saline & $1(6)$ & $0(0)$ & $0(0)$ & $1(1)$ \\
\hline Radio-opaque & $0(0)$ & $7(11)$ & $5(31)$ & $12(13)$ \\
\hline Water & $15(94)$ & $56(89)$ & $11(69)$ & $82(86)$ \\
\hline
\end{tabular}


Table 2 Mean difference ( \pm standard deviation) in intravesical pressure ${ }^{a}$ between transducer groups (cm water)

$M-M$ Microtip-microtip transducer combination, $M-W$ microtip-external water pressure transducer combination, $W-W$ external water-external water pressure transducer combination ${ }^{a}$ Pressures adjusted for baseline differences

\begin{tabular}{llrrr}
\hline Bladder Volume & Maneuver & M-M & M-W & W-W \\
\hline $150 \mathrm{ml}$ & Minimal Valsalva & $-1( \pm 2)$ & $7( \pm 12)$ & $-4( \pm 6)$ \\
& Maximal Valsalva & $-1( \pm 2)$ & $8( \pm 12)$ & $-4( \pm 8)$ \\
& Minimal cough & $-1( \pm 2)$ & $10( \pm 15)$ & $-1( \pm 7)$ \\
& Moderate cough & $-1( \pm 3)$ & $17( \pm 19)$ & $-2( \pm 10)$ \\
& Maximal cough & $-1( \pm 4)$ & $22( \pm 26)$ & $-3( \pm 17)$ \\
$300 \mathrm{ml}$ & Minimal Valsalva & $0( \pm 2)$ & $8( \pm 11)$ & $-5( \pm 6)$ \\
& Maximal Valsalva & $-1( \pm 2)$ & $10( \pm 13)$ & $-5( \pm 7)$ \\
& Minimal cough & $0( \pm 1)$ & $10( \pm 14)$ & $-3( \pm 5)$ \\
& Moderate cough & $-1( \pm 2)$ & $17( \pm 19)$ & $-6( \pm 7)$ \\
& Maximal cough & $-1( \pm 4)$ & $24( \pm 27)$ & $-8( \pm 11)$ \\
\hline
\end{tabular}

numbers, and data were recorded on standardized collection forms that were sent to a central data coordinating center.

The primary goal of the study was to determine the correlation between two transducer types. Thus, a sample size of 60 subjects was necessary to show a minimum correlation of 0.6 with a power of $90 \%$ and a $95 \%$ confidence interval of \pm 0.1 . Using combinations of the same transducers to estimate the variance in the difference between like transducers, we calculated that a minimum of 20 subjects for each like-transducer pair would be needed to provide an estimate with a standard error less than one fourth the standard deviation. The resulting allocation ratio for $\mathrm{M}-\mathrm{M}$, $\mathrm{W}-\mathrm{W}$, and $\mathrm{M}-\mathrm{W}$ combinations was 1:1:3.

Urodynamic parameters were obtained using a standardized protocol agreed on by all participating centers. Each site used 8-French (Fr) Millar microtip transducer catheters (Millar Instruments, Houston, TX, USA) and 8-Fr duallumen water perfusion catheters. There was site-by-site variation in the manufacture of the water perfusion catheter used. Sites 3 and 5 used water perfusion catheters manufactured by Rüsch [Rüsch Manufacturing (UK), Co Armagh, Northern Ireland], while sites 2 and 4 used Medtronic (Medtronic Inc., Minneapolis, MN, USA), and site 1 used Laborie (Laborie Medical Technologies Corp., Toronto, Canada). Prior to the initiation of the study, the urodynamic system at each site was calibrated in a standardized fashion. The microtip transducer was calibrated from 0 to $15 \mathrm{~cm}$ water by immersing the catheter tip in a graduated cylinder $15 \mathrm{~cm}$ high and confirming pressure readings. The external water pressure transducer was calibrated from 0 to $60 \mathrm{~cm}$ water by raising the catheter tip $60 \mathrm{~cm}$ above the transducer and confirming pressure readings.

The transducers were connected to a multichannel urodynamic recorder (Laborie Medical Technologies Corp.; Medtronic Inc.) so that both pressure tracings could be recorded concurrently. Transducer systems were designated as "One" or "Two" based on the connection port used. Microtip transducers were zeroed to atmosphere before placement, while water transducers were zeroed to atmosphere after the transducer module was leveled with the bladder. Both transducers were simultaneously zeroed at the level of the external urethral meatus, placed into the bladder with the pressure sensors rotated $180^{\circ}$ from one another, and stabilized with tape. Subjects were either standing or in a $45^{\circ}$ semireclined position. Room temperature saline, water, or radio-opaque contrast was infused at a rate of $50-60 \mathrm{ml} / \mathrm{min}$, unless otherwise specified due to detrusor overactivity. Subject positioning and type of fluid were determined by a physician's preference and recorded for each subject. Abdominal pressure was measured using either a rectal or a vaginal balloon catheter, with the balloon filled to approximately one third of its unstretched capacity.

Abdominal pressure was equalized with the primary intravesical catheter (the catheter that remained for the clinical portion of the cystometry) as needed to zero detrusor pressure; this procedure did not affect the measurement of intravesical pressure. Baseline vesical pressure was recorded for each catheter at the initiation of filling cystometry. Once the bladder was filled to $150 \mathrm{ml}$, the subject performed two Valsalva efforts and three coughs, allowing the pressure to return to

Table 3 Pearson correlation coefficients between transducer groups

\begin{tabular}{llllllll}
\hline Bladder volume & Groups & & & & \\
\cline { 2 - 7 } & & $\mathrm{M}-\mathrm{M}^{\mathrm{a}}$ & $\mathrm{M}-\mathrm{M}^{\mathrm{b}}$ & $\mathrm{M}-\mathrm{W}^{\mathrm{a}}$ & $\mathrm{M}-\mathrm{W}^{\mathrm{b}}$ & $\mathrm{W}-\mathrm{W}^{\mathrm{a}}$ & $\mathrm{W}^{\mathrm{b}}-\mathrm{W}^{\mathrm{b}}$ \\
\hline $150 \mathrm{ml}$ & Baseline & 0.91 & - & 0.71 & - & 0.74 & - \\
& Cough & 0.99 & 0.99 & 0.87 & 0.89 & 0.97 & 0.96 \\
$300 \mathrm{ml}$ & Valsalva & 0.99 & 0.99 & 0.92 & 0.93 & 0.95 & 0.80 \\
& Baseline & 0.93 & - & 0.66 & - & 0.99 & 0.98 \\
& Cough & 0.99 & 0.99 & 0.88 & 0.99 & 0.94 & 0.97 \\
\hline
\end{tabular}

$M-M$ Microtip-microtip transducer combination, $M-W$ microtip-external water pressure transducer combination, $W-W$ external waterexternal water pressure transducer combination

${ }^{\text {a }}$ Comparison of absolute pressures

${ }^{\mathrm{b}}$ Comparison of pressures after adjustment for baseline differences (comparison of relative increases over baseline) 
baseline between each effort. The Valsalva was first performed with submaximal strain to generate a pressure less than $75 \mathrm{~cm}$ water above baseline and then maximally to a pressure greater than $75 \mathrm{~cm}$ water above baseline. The subject was instructed to cough gently, moderately, and maximally to reach predetermined pressures above baseline (gently, 0-50 cm water; moderately, $50-100 \mathrm{~cm}$ water; maximally, $>100 \mathrm{~cm}$ water). Once testing was completed at $150 \mathrm{ml}$, filling continued to $300 \mathrm{ml}$, at which point, the same series of Valsalva and cough efforts was repeated. Twelve intravesical pressure values were recorded for each catheter, totaling 24 values per catheter pair for each subject. Before analysis, intravesical pressures at baseline were subtracted from those at maximum pressure.

Subjects who had involuntary detrusor contractions with urine loss prior to $150 \mathrm{ml}$ of filling volume were refilled at half of the original filling rate. If subjects were unable to maintain a $150-\mathrm{ml}$ volume of fill for testing, they were excluded.

The primary outcome measure was the difference between maximum intravesical pressure generated for any given effort and baseline intravesical pressure before that effort, as measured by each of the transducers. The mean difference and its standard deviaton were computed between each pair of transducers for each type and strength of maneuver at each volume. When the transducers were of different types, the difference between the pressures obtained by the microtip less the external water transducers was computed; otherwise, the difference between like transducers was computed (transducer One less transducer Two) to estimate variance. Correlation plots were generated, and repeated measures analysis of variance was used to identify mean differences significantly different from 0 . Secondary analyses considered the impact of bladder volume, subject positioning, type of fluid, and clinical characteristics, such as height and weight, on the correlation between transducers. The data analysis team was not masked to the transducer combinations.

\section{Results}

Of the 107 women enrolled, 12 were excluded for detrusor overactivity $(n=8)$, other clinical condition preventing bladder filling to $150 \mathrm{ml}(n=3)$, or stage III or greater prolapse $(n=1)$, leaving 95 subjects for analysis.

Women ranged in age from 28 to 87 years (mean \pm standard deviation $54.2 \pm 13.0$ years) (Table 1). Age, height, weight, and body mass index (BMI) were similar across the groups. Most tests were performed in the semireclined position $(87 \%)$ using sterile water $(86 \%)$. As a result, the effects of patient positioning and fluid medium on intravesical pressure were not studied, given the small numbers in the comparison groups.

As shown in Table 2, pressures from microtip transducers $(\mathrm{M}-\mathrm{M})$ showed the smallest mean differences $(0-1 \mathrm{~cm}$ water), while water transducers (W-W) showed slightly larger mean differences (1-8 cm water). Large mean differences were observed when comparing microtip and water transducers (M-W $8-25 \mathrm{~cm}$ water, all $p$ values $<0.001$ by

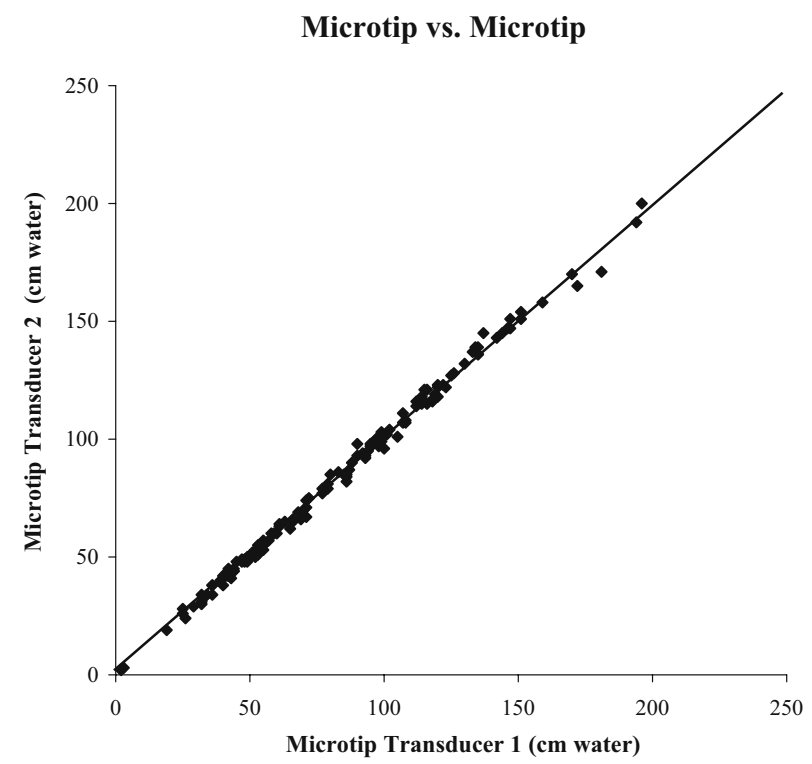

Fig. 1 Correlation plot of microtip vs microtip transducers for Valsalva and cough efforts at all strengths and bladder volumes (150 and $300 \mathrm{ml}$ )

paired $t$ test). The standard deviations of the differences were large between microtip and water transducers (M-W 10 $27 \mathrm{~cm}$ water) compared to catheters of the same type (M-M 1-4 cm water, W-W 5-17 cm water). The mean differences were similar for both coughs and Valsalva efforts within each transducer pairing, regardless of strength of effort or bladder volume.

Given the significant differences seen in the M-W transducer pairings, data were analyzed by site. Sites 1, 3, and 5 demonstrated significant mean differences between

External Transducer vs. External Transducer

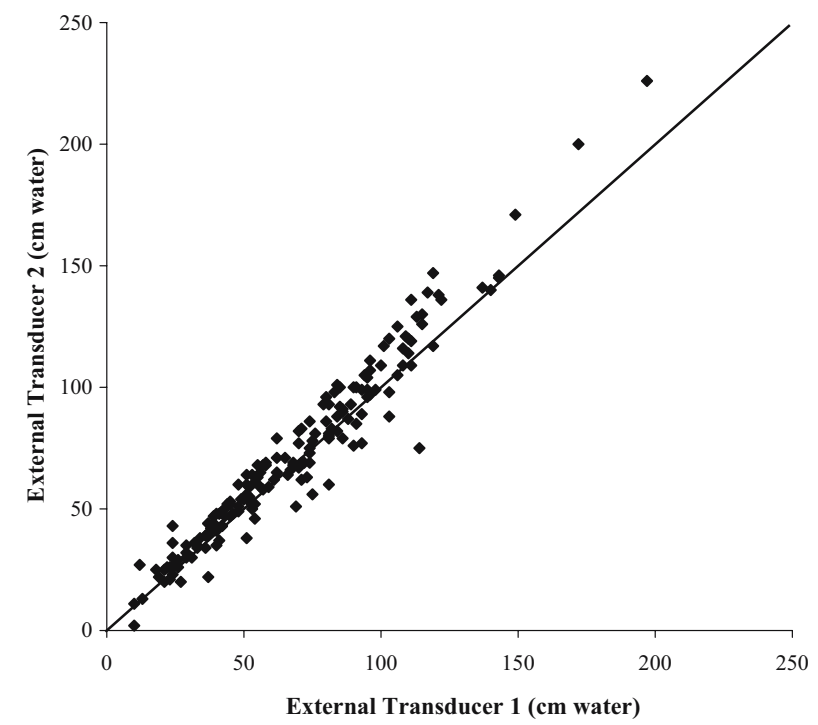

Fig. 2 Correlation plot of water vs water transducers for Valsalva and cough efforts at all strengths and bladder volumes (150 and $300 \mathrm{ml})$ 


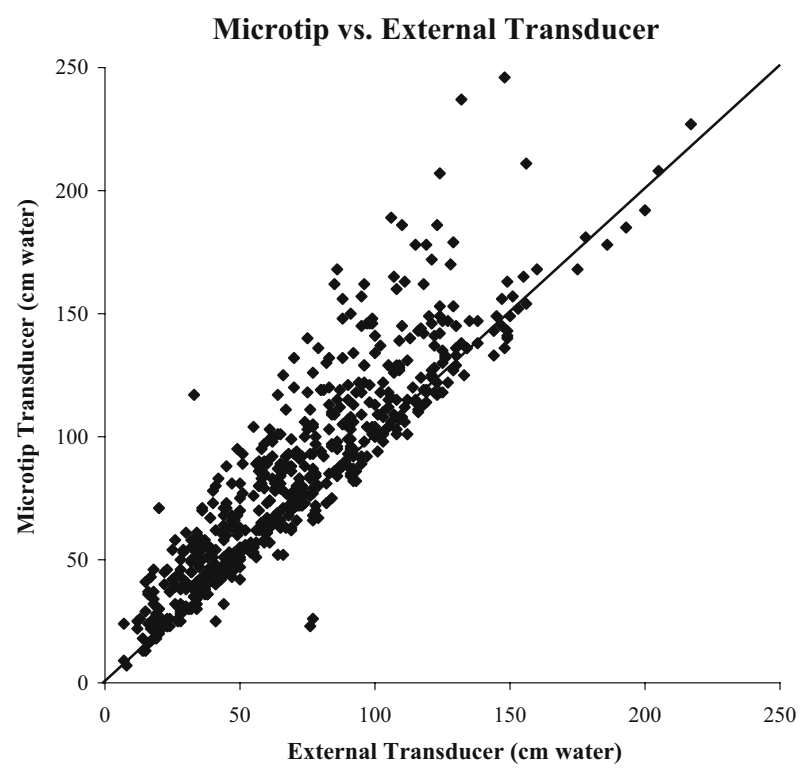

Fig. 3 Correlation plot of microtip vs water transducers for Valsalva and cough efforts at all strengths and bladder volumes (150 and $300 \mathrm{ml})$

microtip and water transducers for most pressure readings ( $0-36 \mathrm{~cm}$ water, $p$ values ranging from $p<0.0001$ to $p=0.3$ ), while sites 2 and 4 did not demonstrate significant differences between microtip and water transducers $(3-7 \mathrm{~cm}$ water, all $p>0.07)$. For $\mathrm{M}-\mathrm{W}$ transducer combinations at sites 1,3 , and 5 , the microtip transducer in general provided higher pressure readings than the water transducer.

Correlation of maximum pressure was consistently high between microtip transducers $\left(\mathrm{M}-\mathrm{M}^{\mathrm{b}} r=0.99\right)$, regardless of Valsalva or cough or bladder volume (Table 3; Fig. 1). Correlation between water transducers was also high $\left(\mathrm{W}-\mathrm{W}^{\mathrm{b}} r=0.96-0.98\right)$ (Table 3; Fig. 2). Correlations were lowest between microtip and water transducers $\left(\mathrm{M}-\mathrm{W}^{\mathrm{b}} r=\right.$ 0.89-0.94) (Table 3; Fig. 3). Bladder volume, age, and BMI were not significantly related to mean intravesical pressure for all catheter combinations.

Correlations were also computed by site. There was little variability to correlation values for like-transducer pairs, regardless of site, cough, or Valsalva maneuver or bladder volume ( $r=0.92$ and higher). For the $\mathrm{M}-\mathrm{W}$ transducer pairs, results were similar to those for mean differences, with sites 1,3 , and 5 showing lower correlation $(r=0.74$ $0.98)$ than sites 2 and $4(r=0.93-0.99)$. Within the M-W combination, correlations were consistently lower for cough $(r=0.74-0.92)$ than for Valsalva $(r=0.93-0.98)$, with the effect again limited to sites 2,4 , and 7 .

\section{Discussion}

For research purposes, especially in the setting of a multicenter study that uses urodynamic measures of intravesical pressure, our data confirm that microtip and water-based transducer catheters should not be used interchangeably. Indeed, the large mean differences and vari- able correlations between microtip and water transducers after cough and Valsalva maneuvers observed in this study have the potential to introduce systematic bias into multicenter urodynamic data. Therefore, careful standardization of urodynamic technique should include standardization of a specific catheter/transducer combination.

Multicenter urodynamic studies pose unique challenges and require special attention to standardization to maintain high-data quality. The microtip transducer universally performed better than the external water transducer with consistently high correlations and low mean differences. There was a much higher degree of variability in the performance of some of the water-based transducer catheters used in this study. Researchers should consider microtip transducers as a first-line catheter for multicenter urodynamic studies using intravesical pressures as outcome measures. However, certain water catheter/transducer systems appear to provide highly reproducible data with a much smaller degree of variability than others.

The large differences in intravesical pressures seen when using a microtip transducer compared to some external water catheter/transducer systems may in part be explained by the inherent difference in the frequency/response of these two systems. In 1987, the ICS Working Party on Urodynamic Equipment reported that the frequency/response of modern transducers used for physiological purposes was more than adequate when pressure measurements were made at the source, as they are with catheter-mounted microtip transducers [8]. This same document notes that the sampling rate for external transducers is considerably slower. Consequently, external water transducers may be less likely to capture the actual peak of the pressure tracing compared to microtip transducers, which are operating at a higher frequency/ response rate. This concern is supported by the finding that the microtip transducers consistently measured a higher intravesical pressure than the external water transducers (Fig. 3). The $45^{\circ}$ line demonstrating equivalent values between the two transducers clearly lies below the vast majority of points, consistent with the microtip transducer recording a higher simultaneous value than the water transducer.

Differences in pressure readings resulting from the difference in frequency/response are particularly pronounced when rapid changes of pressure occur, such as with coughs [8]. As demonstrated in our data, the observed differences between microtip and water transducers were more pronounced with coughs compared to Valsalva efforts at some sites. While these differences between microtip and water transducers were predominantly moderate in scale (i.e., 15 $20 \mathrm{~cm}$ water), some individual differences were noted to be greater than $100 \mathrm{~cm}$ water. These differences likely represent technical difficulties such as the presence of a small air bubble in the water catheter or contact between the microtip catheter diaphragm and the bladder wall.

The ICS Working Party on Urodynamic Equipment also cautioned that if the pressure to be measured was transmitted to the external transducer via a liquid-filled catheter, then the dynamic characteristics of the system would be limited by a multitude of factors, including the length and stiffness of the catheter and tubing, the lumen diameter, and the fluid medi- 
um. These concerns are reflected in the variable performance of the $\mathrm{M}-\mathrm{W}$ transducer pairs in this study. Two sites produced mean differences and correlations similar to those for likepaired transducers, while three other sites showed large mean differences and poorer correlations. One difference between the sites was the manufacturer of the water catheter used. The study protocol standardized the use of a dual-lumen, 8-Fr water catheter but not a particular manufacturer, since this is not usually standardized in multicenter urodynamic trial. Sites 2 and 4 used one type of catheter, while sites 3 and 5 used a second type, and site 1 used a third. This suggests that some water catheters may provide more reliable data than others. Suboptimal catheter performance will likely exacerbate pressure differences seen as a result of frequency/response variations.

It may be argued that poor study protocol and technique at sites 1,3 , and 5 were responsible for the poor performance of the $\mathrm{M}-\mathrm{W}$ transducer pairs. However, poor technique would likely have resulted in an even distribution of the pressure readings above and below the $45^{\circ}$ line. Instead, at each of the three sites, data were uniformly above the $45^{\circ}$ line, with the microtip transducer consistently producing a higher pressure reading than the external transducer. In addition, performance of the $\mathrm{M}-\mathrm{M}$ transducer pairs was excellent and consistent with that at sites 2 and 4 .

It is worth commenting on the difference between theory and practice. In theory, mean differences between transducers of similar type (M-M or W-W) should be 0 if both systems are standardized with regard to calibration, zeroing, placement, and use. In practice, as in this clinical trial, we observed differences. A mean difference close to 0 was seen for the $\mathrm{M}-$ $\mathrm{M}$ combination. For the $\mathrm{W}-\mathrm{W}$ combination, however, there were considerable mean differences, as well as differences for individual efforts between transducers, some as great as $50 \mathrm{~cm}$ water. These large differences likely represent the occurrence of significant artifact despite a standardized protocol and adherence to ICS standards. We believe they speak to the inherent variability of an external transducer system relative to a catheter-mounted system. The remaining variability in the $\mathrm{W}-\mathrm{W}$ combination may be the result of inconsistent performance of specific water catheters as noted above.

The near-perfect degree of correlation observed in the experimental model [7] was not reproduced in our human experiment. One possible explanation for the apparent differences is that the simulated "coughs" generated by manual external compression in the experimental model resulted in a slower rise of pressure over time compared to the human experiment. Thus, the greater frequency response of the microtip transducer was not apparent in the experimental model. Moreover, since external water pressure transducers appear to be influenced by internal catheter diameter and length of tubing, further studies are needed to evaluate how these differences affect pressure recordings. Additionally, no comparisons were made between pressure readings from a typical single vesical catheter and this experimental dual catheter system to verify that the dual catheter design did not inherently alter the functionality of the catheters themselves. Such interaction between two intravesical catheters could hypothetically be responsible for some or all of the pressure differences seen. Finally, the potential impact of radio-opaque dye or patient position on catheter pressures is worthy of further investigation but cannot be commented on here due to small numbers in the comparison groups.

The need for standardization of urodynamic procedures, including catheter and transducer type, has been suggested based on theoretical concerns for several years. However, before this study, the actual impact of transducer type on measurement bias in a clinical setting has never been demonstrated. Given the increased number of collaborative networks performing multicenter trials involving urodynamics, standardization of urodynamic procedures is particularly important. Adoption of reliable, standardized urodynamic techniques has the potential to generate highquality data that could be shared and compared across individuals or networks.

These data are also relevant to the physician in clinical practice. While variation between catheter or transducer types is unlikely for studies performed by a single practitioner, there continues to be significant variation in the catheter and transducer systems used between individuals and groups. Patients seen by more than a single individual or group during the process of their clinical evaluation may have urodynamic studies performed with various systems. These data suggest that if different catheter or transducer types are used, the information generated may not be directly comparable. This limits the confidence that can be placed on such values, especially when the values might otherwise be used to determine clinical management, such as the choice of anti-incontinence procedure.

\section{Conclusions}

Excellent reproducibility was demonstrated with transducers of the same type for intravesical pressures recorded during Valsalva and cough in women without prolapse. However, considerable variability was seen in pressures recorded by different transducers, particularly with certain varieties of catheter manufacturer, indicating that intravesical pressure recordings from microtip and water-based systems are not interchangeable. These findings reinforce the need to adopt standardized urodynamic techniques within multicenter research trials and for practicing clinicians using urodynamic results to guide patient management. A consistent methodology is necessary to obtain high-quality data that could be compared across individuals or networks.

Acknowledgements The authors would like to thank Robert Park, Chairman of the Steering Committee for the PFDN.

This work was supported by grants from the National Institute of Child Health and Human Development (U01 HD41249, U10 HD41268, U10 HD41248, U10 HD41250, U10 HD41261, U10 HD41263, U10 HD41269, and U10 HD41267). 


\section{References}

1. Abrams P, Cardozo L, Fall M et al (2002) The standardisation of terminology of lower urinary tract function: report from the Standardisation Sub-committee of the International Continence Society. Am J Obstet Gynecol 187:116-126

2. Bump RC, Elser DM, Theofrastous JP, McClish DK (1995) Valsalva leak point pressures in women with genuine stress incontinence: reproducibility, effect of catheter caliber, and correlations with other measures of urethral resistance. Continence Program for Women Research Group. Am J Obstet Gynecol 173:551-557

3. Swift SE, Utrie JW (1996) The need for standardization of the Valsalva leak-point pressure. Int Urogynecol J Pelvic Floor Dysfunct 7:227-230

4. Swift SE, Yoon EA (1999) Test-retest reliability of the cough stress test in the evaluation of urinary incontinence. Obstet Gynecol 94:99-102

5. Miklos JR, Sze EH, Karram MM (1995) A critical appraisal of the methods of measuring leak-point pressures in women with stress incontinence. Obstet Gynecol 86:349-352

6. Wang AC, Chen MC (2002) A comparison of urethral pressure profilometry using microtip and double-lumen perfusion catheters in women with genuine stress incontinence. BJOG 109:322-326

7. Hundley AF, Visco AG (2004) Comparison of measurements obtained with microtip and external water pressure transducers. Int Urogynecol J Pelvic Floor Dysfunct 15:276-280

8. Rowan D, James ED, Kramer AE, Sterling AM, Suhel PF (1987) Urodynamic equipment: technical aspects. Produced by the International Continence Society Working Party on Urodynamic Equipment. J Med Eng Technol 11:57-64 